\title{
Sports Biomechanics
}

\section{Influence of saddle setback on knee joint forces in cycling}

\section{Mathieu Menard, Mathieu Domalain, Arnaud Decatoire \& Patrick Lacouture}

To cite this article: Mathieu Menard, Mathieu Domalain, Arnaud Decatoire \& Patrick Lacouture (2018): Influence of saddle setback on knee joint forces in cycling, Sports Biomechanics, DOI: 10.1080/14763141.2018.1466906

To link to this article: https://doi.org/10.1080/14763141.2018.1466906

册Published online: 19 Jun 2018.

Submit your article to this journal $\asymp$

Q View related articles $\llbracket$

View Crossmark data \lceil 


\title{
Influence of saddle setback on knee joint forces in cycling
}

\author{
Mathieu Menard ${ }^{a}, \mathrm{~b}$, Mathieu Domalain ${ }^{\mathrm{b}}$ (D), Arnaud Decatoire ${ }^{\mathrm{b}}$ and Patrick Lacouture ${ }^{\mathrm{b}}$

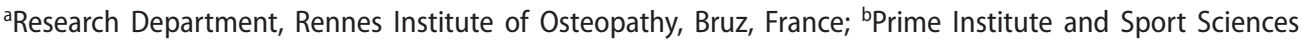 \\ Department, University of Poitiers, Poitiers, France
}

\begin{abstract}
Knee functional disorders are one of the most common lower extremity non-traumatic injuries reported by cyclists. Incorrect bicycle configuration may predispose cyclist to injury but the evidence of an effect of saddle setback on knee pain remains inconclusive. The aim of this study was to determine the effect of saddle setback on knee joint forces during pedalling using a musculoskeletal modelling approach. Ten cyclists were assessed under three saddle setback conditions (range of changes in saddle position $\sim 6 \mathrm{~cm}$ ) while pedalling at a steady power output of $200 \mathrm{~W}$ and cadence of $90 \mathrm{rpm}$. A cycling musculoskeletal model was developed and knee joint forces were estimated using an inverse dynamics method associated with a static optimisation procedure. Our results indicate that moving the saddle forwards was not associated with an increase of patellofemoral joint forces. On the contrary, the tibiofemoral mean and peak compression force were 14 and 15\% higher in the Backward than in the Forward condition, respectively. The peak compression force was related to neither pedal force nor quadriceps muscle force but coincided with the eccentric contraction of knee flexor muscles. These findings should benefit bike fitting practitioners and coaches in the design of specific training/rehabilitation protocols.
\end{abstract}

\section{ARTICLE HISTORY}

Received 17 February 2017 Accepted 13 April 2018

\section{KEYWORDS}

Biomechanics; knee overuse injuries; musculoskeletal modelling; tibiofemoral forces; bike fitting

\section{Introduction}

Together with low back pain, knee functional disorders are one of the most common lower extremity non-traumatic injuries reported by well-trained cyclists (about 50\% of injured cyclists) (Clarsen, Krosshaug, \& Bahr, 2010). Those injuries include tendinopathies (patellar tendinitis, iliotibial band syndrome, etc.) and cartilage/bone degradation (chondromalacia patella, meniscus damage, etc.) that account for $\sim 32 \%$ of clinical diagnosis of the overuse injuries (De Bernardo, Barrios, Vera, Laíz, \& Hadala, 2012). Some authors suggested that an incorrect bicycle configuration may predispose cyclists to knee joint overuse injury (Asplund \& St Pierre, 2004; Callaghan, 2005; Wanich, Hodgkins, Columbier, Muraski, $\&$ Kennedy, 2007). In particular, large changes in saddle height (>3\% of leg length) were associated with increases in knee joint forces (Bini, Hume, \& Croft, 2011 ; Ericson \& Nisell, 1986; Tamborindeguy \& Bini, 2011). The influence of saddle setback is more unclear, only a 
few opinion articles (Asplund \& St Pierre, 2004; Callaghan, 2005; Holmes, Pruitt, \& Whalen, 1994; Silberman, Webner, Collina, \& Shiple, 2005) suggested a link with anterior knee pain. However, the prevalence of knee joint injuries is not higher in track cyclists and triathletes than in road cyclists (De Bernardo et al., 2012; Gosling, Gabbe, \& Forbes, 2008) even if they tend to have their saddle more forward (Ricard, Hills-Meyer, Miller, \& Michael, 2006).

Saddle setback is defined as the distance between the rear of saddle and the axis of the chainset (de Vey Mestdagh, 1998), which implies that an increased saddle setback involves a more backward position of the saddle. Riding with a saddle position more forward increased knee joint angle, lower limb joint mechanical work and rectus femoris activation (Bini, Hume, Lanferdini, \& Vaz, 2014) while changes in fore-aft sitting position (the saddle being stationary) did not substantially affect patellofemoral and tibiofemoral compression forces (Bini, Hume, Lanferdini, \& Vaz, 2013).

Results of Bini et al. (2013) were based on an inverse dynamics procedure associated with a geometric model of the knee joint. Musculoskeletal modelling accounts for co-contraction of all muscles that cross the joint influence of neighbouring joints and muscle mechanics (e.g. muscle force-length relationship). It may lead to a more realistic estimation of joint forces (Blache, Creveaux, Dumas, Chèze, \& Rogowski, 2016; Delp et al., 2007; Marshall \& McNair, 2013; Schwameder, Lindenhofer, \& Müller, 2005). The aim of this study was to determine the effect of saddle setback on knee joint forces during pedalling using a musculoskeletal modelling approach. We hypothesised that a smaller saddle setback would lead to an increase in patellofemoral force (based on epidemiological studies) and a decrease in tibiofemoral force.

\section{Methods}

\subsection{Participants}

Ten club competitor cyclists (Ansley \& Cangley, 2009) without history of knee pain or injury volunteered to participate in the study $(30.9 \pm 8.6$ years, $175.1 \pm 0.1 \mathrm{~cm}, 65.2 \pm 8.3 \mathrm{~kg})$. They had $8.5 \pm 6.8$ years of experience in competition and their average weekly training volume was $4.0 \pm 0.8 \mathrm{~h}$. Participants were informed of the procedures, methods, benefits and possible risks involved in the study before their written consent was obtained. The study protocol was approved by the University of Poitiers Ethics Committee and met the requirements of the Declaration of Helsinki for research on human beings.

\subsection{Experimental set-up and procedures}

A stationary cycle ergometer SRM Indoor Trainer (SRM, Schoberer, Germany) was instrumented to conduct the experiment. The seat and handlebar position were fully adjustable. Three six-load component force sensors (Sensix, Poitiers, France) were integrated into the two pedals and seat post. According to the manufacturer, this sensor had a maximum $1 \%$ error in each direction (percentage related to the whole working range of the transducer and including linearity and hysteresis error). The sensors were calibrated before recording of each new participant, following the recommendation of the manufacturer. Digital encoders (Scancon 2MCA, Hillerød, Denmark, resolution $0.018^{\circ}$ ) were synchronised with the pedal sensors in order to get the instantaneous pedal-to-crank angle and then the torque applied to the crank. 
A 20-camera motion analysis system (Vicon Motion Analysis Inc., Oxford, UK) was used to compute three-dimensional kinematics. The marker set was based on a previous study (Hamner, Seth, \& Delp, 2010). Specific software was developed to synchronise, capture $(200 \mathrm{~Hz})$, process and save the experimental data (Sensix, Poitiers, France).

\subsection{Musculoskeletal modelling}

Marker data and pedal force-moments served as input of the model for the computation of muscle forces. A musculoskeletal model including 13 segments, 31 degrees of freedom, 86 muscles was developed based on a full-body model originally developed for gait and running activities (Hamner, Seth, \& Delp, 2010). A few modifications were made to adapt the model to the specific analysis of knee joint forces in cycling: (1) the patellofemoral joint was added based on a previous study (Arnold, Ward, Lieber, \& Delp, 2010), (2) knee, hip and ankle joint ranges of motion were increased to correspond to the pedalling task (for example, maximal knee flexion angle was increased from $-110^{\circ}$ to $\left.-130^{\circ}\right),(3)$ an ellipsoidal wrapping surface was included in the inferior extremity of the femur so that knee extensor moment arms remained consistent with published values (Arnold et al., 2010; Spoor \& Van Leeuwen, 1992) in the added ranges of motion, (4) the metatarsophalangeal joint was locked to be more truthful with the use of rigid soles and clip-less pedals, (5) estimations of maximum isometric force included in the original model had been shown to be unrealistically low and were therefore uniformly increased $50 \%$ for muscles as in previous studies (Arnold et al., 2010; Raabe \& Chaudhari, 2016).

\subsection{Experimental protocol}

In order to focus on the influence of saddle setback, the set-up of all other settings was standardised. Crank arm length was identical $(175 \mathrm{~mm})$ among all participants and conditions. Pedals cleats were adjusted for each participant such as the head of the first metatarsal was positioned directly above the pedal spindle. Handlebar height and reach were defined based on individual anthropometric measurements of arm/torso lengths (de Vey Mestdagh, 1998). Three saddle setback conditions were compared: a Recommended setback condition, a Backward (10\% more backward) and a Forward (10\% more forward) setback conditions. The Recommended position was based on anthropometric measurements of inside leg length (the barefoot distance between the ground and the pubis) and the upper leg length (the distance between the sacral apex and the anterior part of the patella). From these two measurements, saddle height and setback were adjusted following recommendations of de Vey Mestdagh (1998) and previously used by Menard et al. (2016). This Recommended position was not assumed the optimal position for all cyclists, but aimed at standardising the position (i.e. a common reference saddle position for all cyclists). The actual sitting position (i.e. horizontal distance between centre of pressure location on the saddle and the chainset axis centre) was recorded using a force sensor and results were reported in Menard et al. (2016). For Backward and Forward conditions, saddle vertical height was adjusted to exclude the potential influence of a non-constant distance between the centre of the chainset and the midpoint of the top of the saddle (Figure 1). The adjustment of saddle height preserved a constant distance between the saddle and the centre of the chainset resulting in a slight rotation of the whole cyclist's body around the chainset instead of moving him closer to/ 


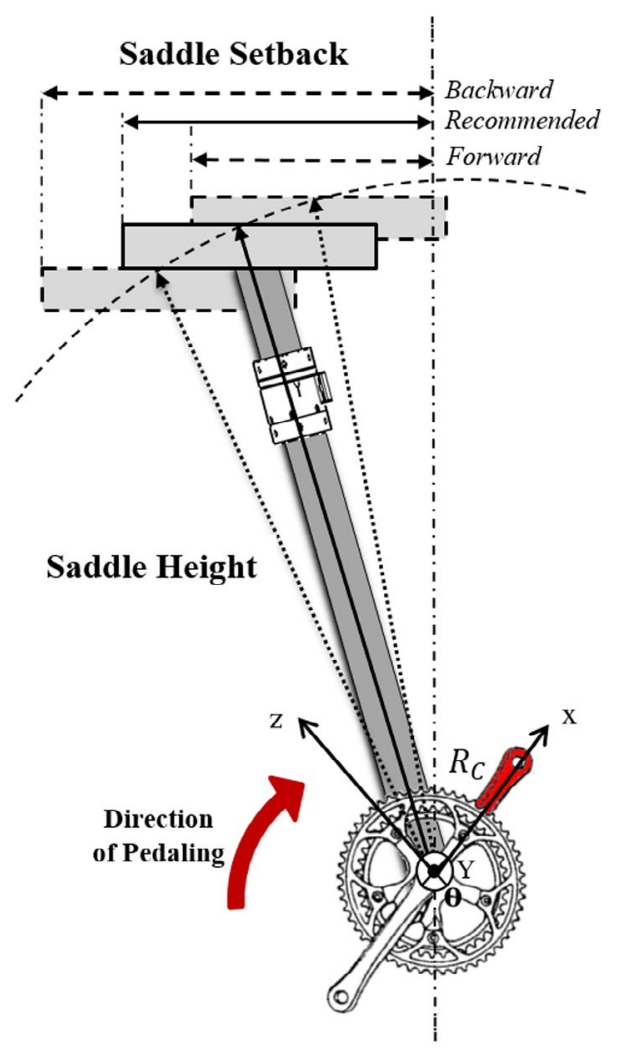

Figure 1. Representation of saddle height and setback measurements.

further away from it. In that sense it mimics a change of seat tube angle (Caddy, Times, \& Gordon, 2016; Leirdal \& Ettema, 2011). Theoretically, this would allow to conserve the joint angles and muscle lengths standardised according to anthropometrics-based recommendations (de Vey Mestdagh, 1998). Handlebar height/reach was adjusted in order to keep these values constants across all conditions. The evaluation was preceded by a 10 -min free warm up with the Recommended position. Then for each of the three setback conditions, participants were instructed to perform a three-minute trial while keeping cadence ( $90 \mathrm{rpm}$ ) and power $(200 \mathrm{~W})$ constant in order to reflect the protocol used by previous relevant studies (Neptune \& Kautz, 2000; Price \& Donne, 1997). Continuous visual feedback about cadence and power was provided on the screen of the bicycle computer. A minimum of three min of active recovery rest at freely chosen power and cadence between trials was given to the participants to avoid any effect of fatigue. Each condition was completed once and the order of trials was randomised.

\subsection{Data analysis}

The first two minutes of each trial allowed the participant to accommodate with the new saddle position and get back to a steady state. Only the last minute was recorded and served 
as input of the simulation. Thereafter outcomes data (muscle and joint forces) were averaged over 30 consecutive crank cycles.

The estimation of knee joint forces resulted from Opensim calculation steps (Delp et al., 2007): (1) scaling, (2) inverse kinematics, (3) inverse dynamics, (4) static optimisation and (5) joint reaction analysis. First the model (segment lengths, mass distribution, muscle attachment sites, etc.) was scaled to match the participant's anthropometry based on experimentally measured markers placed on anatomical landmarks. Participants performed functional movements (circumduction) to estimate the location of joint centres at the hip, knee and ankle (Ehrig et al., 2011). Secondly, joint angles were calculated through inverse kinematics: a global optimisation problem was solved that minimised the differences between experimentally measured markers trajectories and the corresponding markers placed on the theoretical model. Muscle forces (as well as muscle length and velocity) were calculated through static optimisation, minimising a physiological criterion (sum of muscles activations squared) (Demers, Pal, \& Delp, 2014). Finally, bone-to-bone joint forces were calculated using the Joint Reaction analysis in OpenSim (Steele, DeMers, Schwartz, $\&$ Delp, 2012). The analysis was focused on the right knee joints only: the tibiofemoral and the patellofemoral joints. Tibiofemoral forces and patellofemoral forces were expressed in their respective joint reference system (child segment) and normalised to crank angle. The mean and peak of the three components of each knee joint forces were calculated.

\subsection{Statistical analysis}

Data were first tested for normality with the Shapiro Wilk test. The normality of the distributions was respected. To test the effect of saddle setback on knee joint forces, a one-way analysis of variance (ANOVA) with repeated measures was performed on peak and mean of all components of the knee joint forces as well as on effective pedal force (perpendicular to the long axis of the crank), knee angle and knee muscle forces at the time of tibiofemoral peak compression force. Where relevant, the pairwise comparison were rated via Cohen's effect sizes (ES) as small $(0.25<\mathrm{ES}<0.49)$, moderate $(0.5<\mathrm{ES}<1)$ and large $(\mathrm{ES}>1)$ (Rhea, 2004). All data are presented as mean (standard deviation) and alpha (level of significance) was set to 0.05 . All statistical analyses were performed using Statistica (StatSoft, Inc.; Tulsa, OK).

\section{Results}

Across participants and conditions average values of power and frequency were $194.5 \pm 11.9 \mathrm{~W}$ and $90.9 \pm 1.3 \mathrm{rpm}$, respectively.

Saddle setback had no effect on the three components (X, Y, Z) of patellofemoral forces. More precisely, there was no significant effect of saddle setback on the mean $(p=0.338$, $p=0.365, p=0.386$, respectively) and the peak $(p=0.436, p=0.826, p=0.592$, respectively) of patellofemoral forces. The peak compression force (X component) occurred at approximately $40^{\circ}$ of crank angle $(1,395.0 \pm 399.7 \mathrm{~N}$, mean of the three conditions) (Figure 2 , right).

There was a significant effect of setback condition on the mean and peak compression ( $\mathrm{Y}$ component) of the tibiofemoral force ( $p<0.05$ for both). The pairwise comparison further detailed that in the Backward condition, the mean of the tibiofemoral compression force $(274.8 \pm 112.9 \mathrm{~N})$ was significantly different from the Forward condition $(248.1 \pm 113.9 \mathrm{~N})$ 

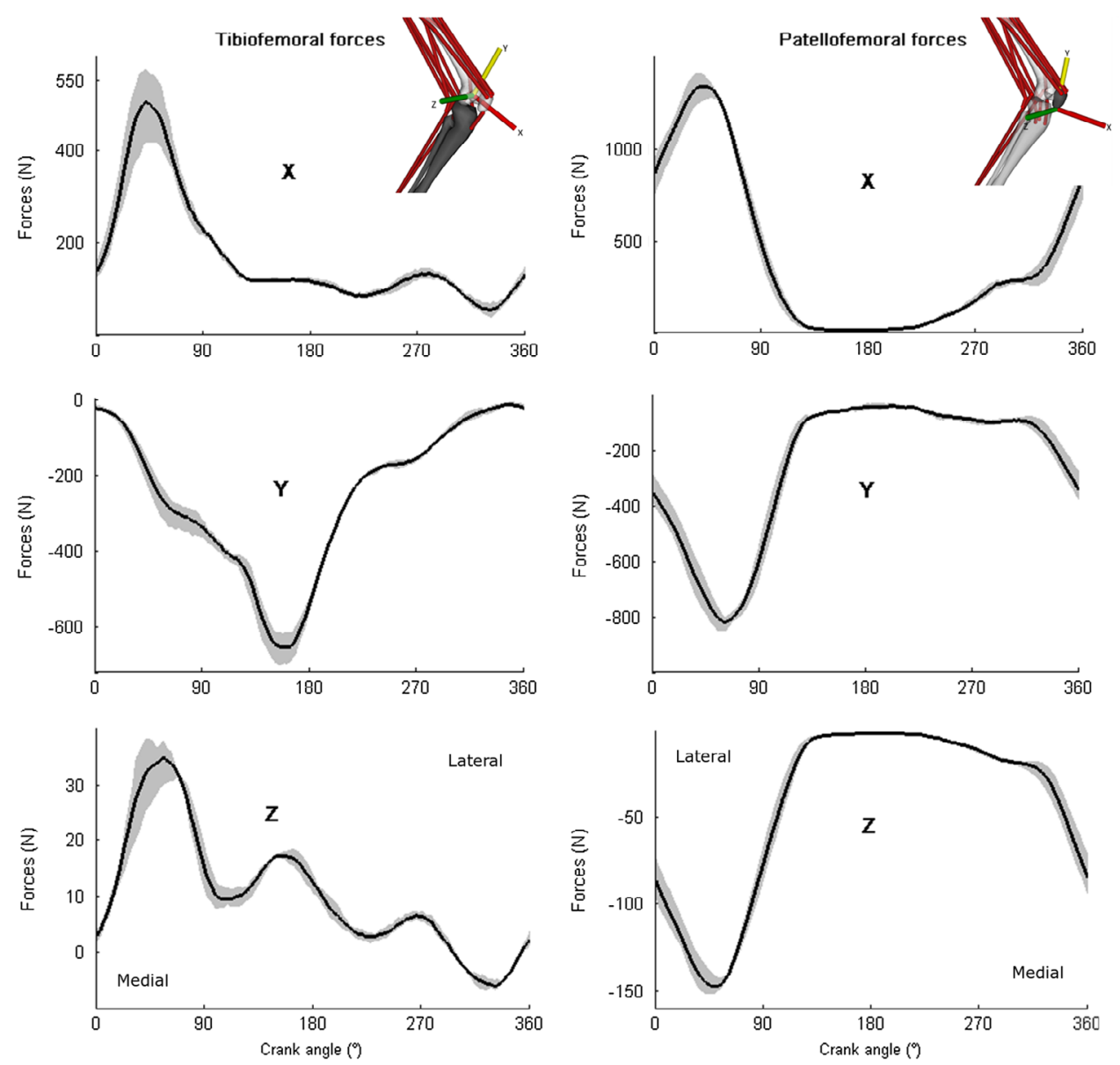

Figure 2. Tibiofemoral and patellofemoral joint forces expressed in their respective joint coordinate systems (solid black line represents the mean across all participants and conditions and grey area the inter-condition standard deviation).

and associated with a small effect size $(p=0.004$, ES $=0.24)$. Similarly, in the Backward condition, the tibiofemoral peak compression force $(732.9 \pm 280.5 \mathrm{~N})$ was significantly different from the Forward condition $(637.1 \pm 260.2 \mathrm{~N})$ and associated with a small effect size $(p=0.002$, ES $=0.35)$. In other words, the mean and peak of tibiofemoral compression force were $14 \%$ and $15 \%$ higher in the Backward than in the Forward condition, respectively. Oppositely, there was no effect of saddle setback on the mean and the peak of the two components $\mathrm{X}$ and $\mathrm{Z}$ of tibiofemoral forces ( $p>0.05$ for both) (Figure 2, left).

The time of peak joint force occurred at approximately $160^{\circ}\left(157 \pm 1^{\circ}\right)$ and there was no effect of setback on this timing. The effect of saddle setback on knee joint angle and knee flexor muscle force was significant and associated with small effect size $(p=0.002$, ES $=0.41$ and $p<0.01$, ES $=0.46$, respectively) at the time of peak tibiofemoral compression force (Figure 3). These results (group mean \pm SD) are detailed in Table 1. Particularly, in the Backward position, knee joint angle was more extended and knee flexor muscle force was higher than in the Forward position. 
(a)

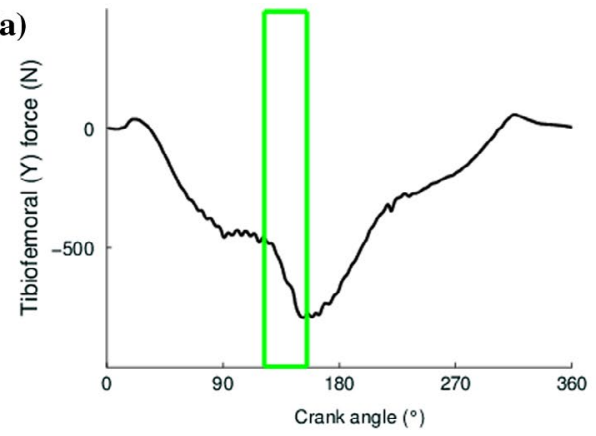

(c)

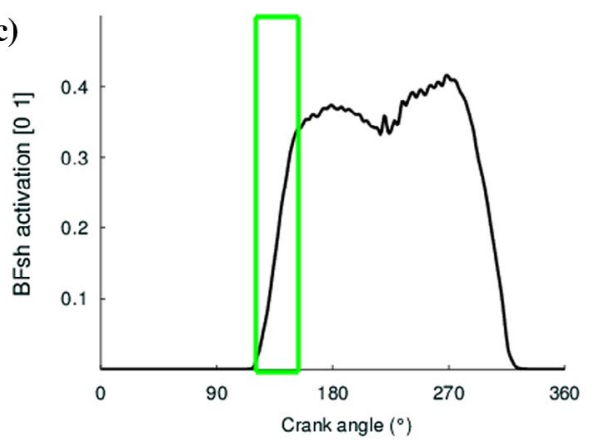

(e)

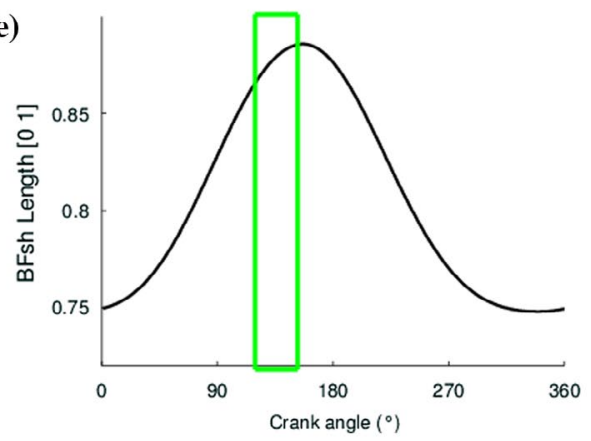

(b)

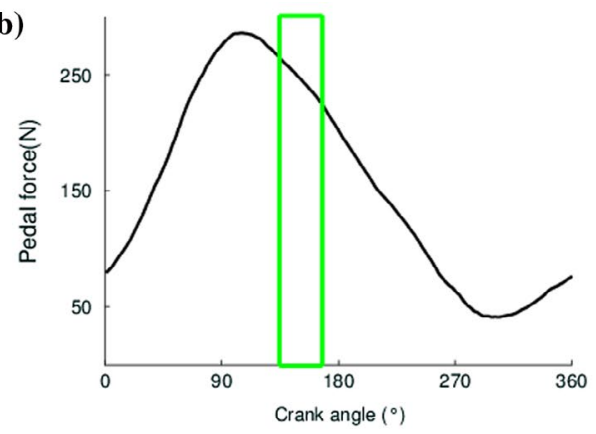

(d)

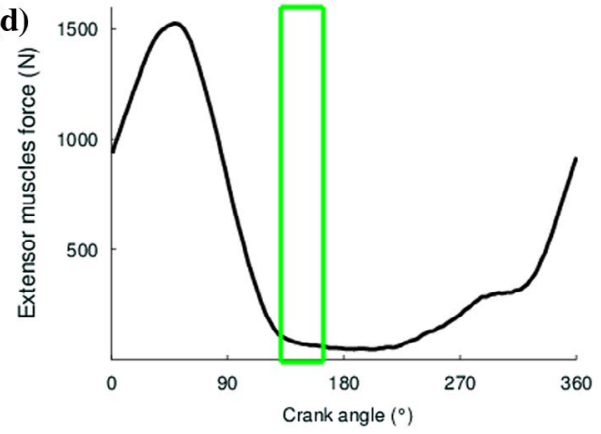

(f)

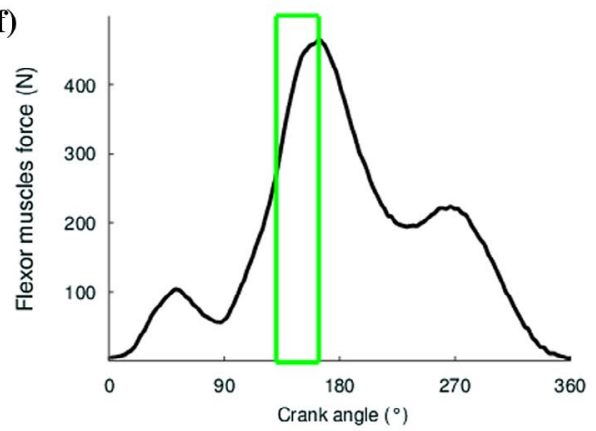

Figure 3. (a) Tibiofemoral compression force estimated through musculoskeletal modelling and inverse dynamics (ID), (b) pedal force, (c) biceps femoral short head (BFsh) activation, (d) knee extensors force, (e) BFsH length and (f) knee flexors force.

Notes: Illustrations represent the mean across all pedalling cycles from one cyclist in the Recommended condition. 0 and $360^{\circ}$ correspond to position when pedal is in top position, and $180^{\circ}$ crank angle to pedal bottom position. The vertical green area highlights the eccentric contraction of knee flexor muscles that occurs in the same time as the peak tibiofemoral compression force.

\section{Discussion and implications}

The purpose of this study was to investigate the influence of saddle setback on knee joint forces when cycling at a given power and cadence. The main finding of this study was that moving the saddle more backward increased tibiofemoral compression force. In addition, peak compression force occurred simultaneously with the eccentric contraction of knee flexor muscles, which also coincided with the slowdown of knee extension. 
Table 1. Mean \pm SD of normalised pedal force, crank angle, extensor/flexor muscle force and knee angle at the time of normalised peak tibiofemoral compression force ( $p$-value and Cohen's effect sizes ES).

\begin{tabular}{lccccc}
\hline Variable & Backward & Recommend d & Forward & $p$ value & ES \\
\hline Peak tibiofemoral compression force $(N)$ & $732.9 \pm 280.5$ & $666.5 \pm 267.1$ & $637.1 \pm 260.2^{*}$ & 0.002 & 0.35 \\
Pedal force $(N)$ & $48.5 \pm 43.1$ & $52.1 \pm 37.0$ & $41.4 \pm 22.5$ & 0.060 & 0.20 \\
Crank angle $\left({ }^{\circ}\right)$ & $158.0 \pm 9.4$ & $159.5 \pm 9.3$ & $156.4 \pm 10.7$ & 0.066 & 0.15 \\
Extensor muscle force $(N)$ & $68.8 \pm 142.0$ & $62.6 \pm 97.8$ & $81.2 \pm 91.4$ & 0.073 & 0.10 \\
Flexor muscle force $(N)$ & $538.3 \pm 242.2$ & $451.9 \pm 198.2$ & $437.6 \pm 247.9^{*}$ & $<0.001$ & 0.41 \\
Knee extension angle $\left({ }^{\circ}\right)$ & $-23.6 \pm 8.9$ & $-26.0 \pm 9.9$ & $-27.3 \pm 6.9^{*}$ & 0.002 & 0.46 \\
\hline
\end{tabular}

${ }^{*} p<0.05$ significant different from Backward.

On the contrary, our results did not reveal any significant influence of saddle setback (in the $\sim 6 \mathrm{~cm}$ range tested) on the patellofemoral forces. This last finding differs from previous epidemiological studies and bike fitting advices (Asplund \& St Pierre, 2004; Holmes et al., 1994; Silberman et al., 2005) that suggested that moving the saddle too forward may increase patellofemoral joint force tied to the prevalence of knee anterior pain. These results are nevertheless consistent with a previous study that found that a forward position of the cyclists, remaining the saddle fixed, did not substantially affect patellofemoral forces (Bini et al., 2013). Probably, the interaction of saddle setback with other bicycle set-up parameters such as crank length or cleat position would still need to be investigated in order to better identify the underlined mechanism responsible for anterior knee pain in cycling.

An increase of $15 \%$ in tibiofemoral compression force was found from the Forward to the Backward condition ( $\sim 6 \mathrm{~cm}$ total change in saddle setback). Bini et al. (2013) found no significant effect on tibiofemoral compression forces. This apparent discrepancy may originate from using a different protocol. Bini et al. (2013) addressed participants' movement on their saddle, which remained stationary, while in this experiment the saddle was moved as a bike-fitting context. Therefore, the overall posture change was greater in this study. In addition, in the present study saddle height was compensated to remain constant beside the change of saddle setback. This relates to the notion of a constant seat tube angle and could explain the different results as well.

An incorrect saddle position (too backward) that increases tibiofemoral compression force may be prejudicial for tibial articular cartilage degradation and menisci damage (Neptune \& Kautz, 2000; Willy et al., 2015). Alongside competitive cycling, it has been shown that pedalling is a common rehabilitation exercise used in order to mobilise joints and increase muscle volume deficiency (Hunt, Sanderson, Moffet, \& Timothy Inglis, 2003; Johnston, 2007). These findings reinforce previous recommendations on bicycle configuration in rehabilitation exercises (Neptune \& Kautz, 2000; Tamborindeguy \& Bini, 2011).

In an attempt to identify the underlying mechanisms responsible for the peak tibiofemoral compression force, pedal force, knee kinematics and knee muscle force/length were investigated as well. We analysed the bone-to-bone tibiofemoral joint forces (computed with musculoskeletal modelling) that include the effect of muscle activity. Previous studies analysed net interaction tibiofemoral joint forces (computed with inverse dynamics) and reported a link between peak compression joint force and pedal force (Ericson \& Nisell, 1986; Ruby, Hull, \& Hawkins, 1992). However, our results showed that this peak was linked to neither pedal peak force (Figure 3(b) nor knee extensor muscles peak force (Figure 3(d) which are the main contributors to the pedalling movement (Ericson \& Nisell, 1986). This 
peak arises later, slightly before $180^{\circ}$, as it can also be seen in figure 4 of Neptune \& Kautz (2000).

Our study shows that peak tibiofemoral compression force corresponded to the knee flexors peak force (Figure 3(f). More precisely, the onset of peak force matched the onset of biceps femoris short head activation (Figure 3(c). At this time, biceps femoris short head was acting eccentrically - the muscle was active (Figure 3(c) while its length was still increasing (Figure 3(e). This was most likely to slow down the extension of the knee at the beginning of this transition phase (Sanderson \& Amoroso, 2009). Increasing saddle setback significantly increased hamstrings peak force. The increased hamstrings muscle forces can be further explained by the fact that the knee joint was more extended in the Backward than in the Forward condition leading to a detrimentally decreased flexor moment arm (Spoor \& van Leeuwen, 1992).

This result not only underlines the important contribution of the hamstrings on the tibiofemoral compression force but also raised clinical interrogations regarding the risk of muscle injury. The epidemiological study of De Bernardo et al. (2012) highlighted that $26.4 \%$ of knee injuries in cycling are overuse muscle pathologies and that, among them, hamstrings $(\sim 11 \%)$ are more affected than the quadriceps muscle $(\sim 7 \%)$. De Bernardo et al. (2012) further highlighted that overuse muscle pathologies such as delayed onset muscle soreness (Brockett, Morgan, \& Proske, 2001) are not enough reported and analysed in the cycling literature. Therefore, even if not yet extensively documented, muscle fibre damage exists in cycling and an increasing saddle setback is likely to exacerbate its potential risk. The combination of eccentric contraction and small range of motion of the hip joint observed in cycling (De Bernardo et al., 2012) may explain the development of shortening of some muscles (psoas and hamstrings) in the long term that may cause recurrent pain and is detrimental from a performance point of view.

There were several limitations to this study. First, personalisation of the model is limited to the scaling of a generic model that approximates participant's anthropometry; using medical imaging would help better account for individual's specific geometry and muscle parameters (Gerus et al., 2013). Also, the knee joint model used in this study does not perfectly represent the complex behaviour of the human patellofemoral and tibiofemoral joints; this may affect the estimated joint forces, even in a seemingly simple movement such as pedalling. The optimisation criterion used in this study is particularly suitable for sub-maximal steady-state tasks (Lin, Dorn, Schache, \& Pandy, 2012; Prilutsky \& Zatsiorsky, 2002) but it has not been extensively tested in cycling. The impact of using another criterion on simulation outcomes still remains to be analysed. Finally, given the limited sample size and the specific population tested (club competitor male cyclists), caution should be paid before generalising these results.

The validation of our musculoskeletal modelling approach is challenging and suffers the lack of in vivo data of joint forces. Firstly, before the interpretation of knee joint forces, we ensured that intermediate results of joint angles and joint moments compared well with data from previous studies (Bini et al., 2016). Secondly, simulated muscle activations were qualitatively evaluated against experimental electromyographic (EMG) measurements collected on all participants using surface electrodes (Delsys, Boston, MA, USA) from six representative muscles: gluteus maximus, rectus femoris, biceps femoris, vastus medialis, soleus and tibialis anterior. Overall, simulated temporal patterns of activations showed good agreement with experimental data (Figure 4), this argues in favour of accurate estimations 

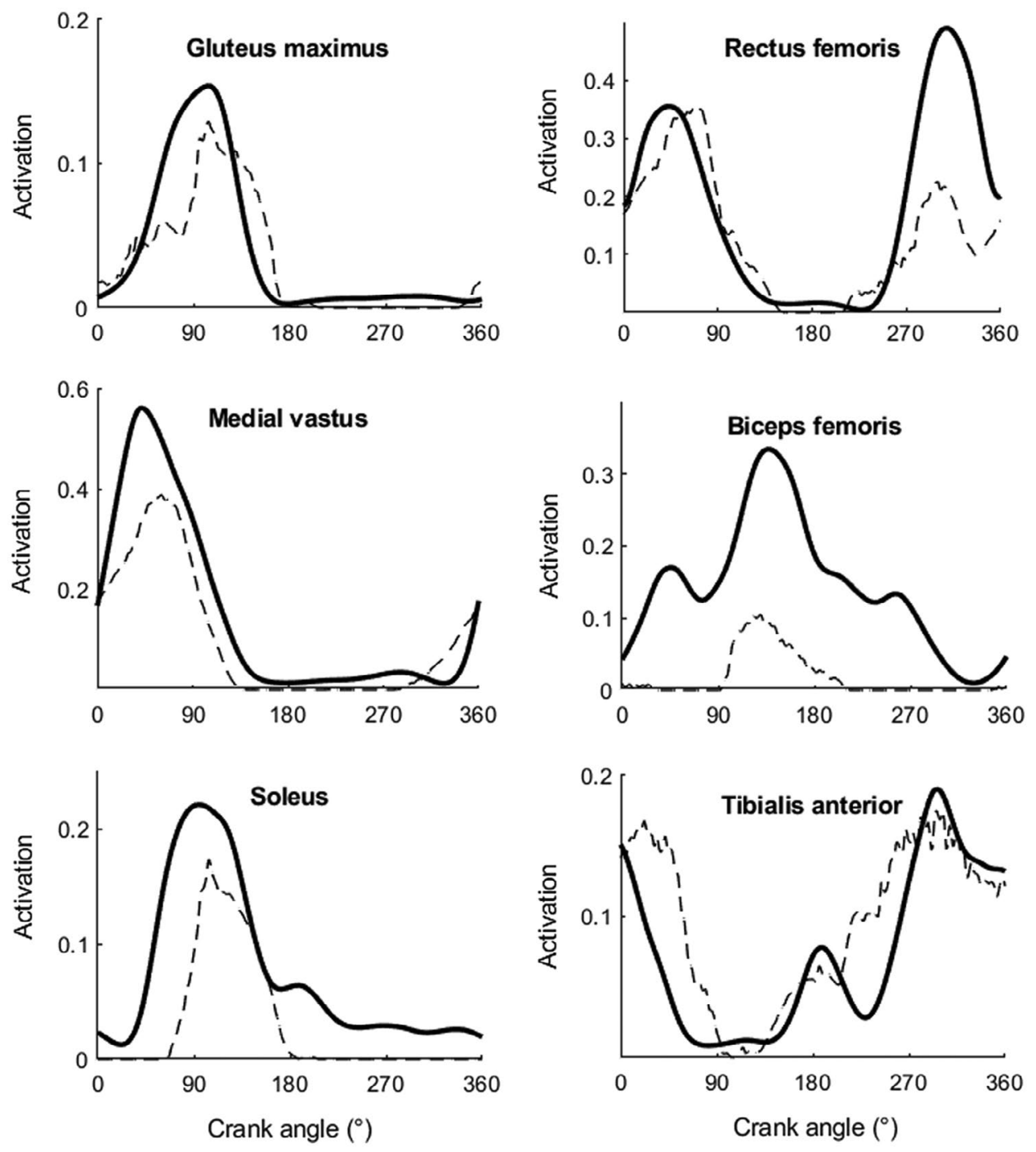

Figure 4. Comparison of muscle activation estimated from static optimisation (dotted line) with experimental EMG (black line).

Note: Example from one participant of one condition (Recommended) of average across 30 crank cycles $\left(0-360^{\circ}\right)$

of muscle activations and subsequent joint forces. Finally, conclusions should be drawn with caution as the effects observed were an acute response to an intervention and a short cycling time in a laboratory.

The present study underlies the difficult compromise between performance and health and reflects the complexity of defining an optimal saddle position and establishing bikefitting guidelines.

\section{Conclusion}

The findings of this study indicate that moving the saddle more forward was not associated with an increase of patellofemoral and tibiofemoral forces. On the contrary, moving the 
saddle more backward may be more detrimental as it leads to higher tibiofemoral compression forces. More generally, this study brings new insights on the underlying mechanisms of tibiofemoral force in cycling that are of importance for a safe training and for rehabilitation protocols.

\section{Acknowledgements}

We thank the athletes for their enthusiastic participation, especially the members of the CRITT Sports Loisirs of Chatellerault. This work has been sponsored by the French government research program 'Investissements d'Avenir' through the Robotex Equipment of Excellence (ANR-10-EQPX-44).

\section{Disclosure statement}

No potential conflict of interest was reported by the authors.

\section{Funding}

This work was supported by the Agence Nationale de la Recherche [grant number ANR-10-EQPX-44].

\section{ORCID}

Mathieu Domalain (D) http://orcid.org/0000-0002-4518-8479

\section{References}

Ansley, L., \& Cangley, P. (2009). Determinants of 'optimal' cadence during cycling. European Journal of Sport Science, 9, 61-85. doi:10.1080/17461390802684325

Arnold, E. M., Ward, S. R., Lieber, R. L., \& Delp, S. L. (2010). A model of the lower limb for analysis of human movement. Annals of Biomedical Engineering, 38, 269-279. doi:10.1007/s10439-009-9852-5

Asplund, C., \& St Pierre, P. (2004). Knee pain and bicycling. The Physician and Sports Medicine, 32(4), 23-30. doi:10.3810/psm.2004.04.201

Bini, R. R., Hume, P. A., \& Croft, J. L. (2011). Effects of bicycle saddle height on knee injury risk and cycling performance. Sports Medicine, 41(6), 463-476. doi:10.2165/11588740-000000000-00000

Bini, R. R., Hume, P. A., Lanferdini, F., \& Vaz, M. (2013). Effects of moving forward or backward on the saddle on knee joint forces during cycling. Physical Therapy in Sport, 14, 23-27. doi:10.1016/j. ptsp.2012.02.003

Bini, R. R., Hume, P. A., Lanferdini, F., \& Vaz, M. (2014). Effects of body positions on the saddle on pedalling technique for cyclists and triathletes. European Journal of Sport Science, 14, 413-420. doi:10.1080/17461391.2012.708792

Bini, R. R., Dagnese, F., Rocha, E., Silveira, M. C., Carpes, F. P., \& Mota, C. B. (2016). Three-dimensional kinematics of competitive and recreational cyclists across different workloads during cycling. European Journal of Sport Science, 16, 553-559. doi:10.1080/17461391.2015.1135984

Blache, Y., Creveaux, T., Dumas, R., Chèze, L., \& Rogowski, I. (2016). Glenohumeral contact force during flat and topspin tennis forehand drives. Sports Biomechanics, 16, 127-142. doi:10.1080/1 4763141.2016.1216585

Brockett, C. L., Morgan, D. L., \& Proske, U. (2001). Human hamstring muscles adapt to eccentric exercise by changing optimum length. Medicine and Science in Sports and Exercise, 33, 783-790. doi:10.1097/00005768-200105000-00017

Caddy, O., Times, M. A., \& Gordon, D. (2016). Effects of saddle angle on heavy intensity time trial cycling: Implications of the UCI rule 1.3.014. Journal of Science and Cycling, 5, 18-25. 
Callaghan, M. (2005). Lower body problems and injury in cycling. Journal of Bodywork and Movement Therapies, 9, 226-236. doi:10.1016/j.jbmt.2005.01.007

Clarsen, B., Krosshaug, T., \& Bahr, R. (2010). Overuse injuries in professional road cyclists. The American Journal of Sports Medicine, 38, 2494-2501. doi:10.1177/0363546510376816

De Bernardo, N., Barrios, C., Vera, P., Laíz, C., \& Hadala, M. (2012). Incidence and risk for traumatic and overuse injuries in top-level road cyclists. Journal of Sports Sciences, 30, 1047-1053. doi:10.1 080/02640414.2012.687112

de Vey Mestdagh, K. (1998). Personal perspective in search of an optimum cycling posture. Applied Ergonomics, 29, 325-334. doi:10.1016/S0003-6870(97)00080-X

Delp, SL, Anderson, F. C., Arnold, A. S., Loan, P., Habib, A., John, C. T., ... Thelen, D. G. (2007). OpenSim: Open-source software to create and analyze dynamic simulations of movement. IEEE Transactions on Biomedical Engineering, 54, 1940-1950. doi:10.1109/TBME.2007.901024

Demers, M. S., Pal, S., \& Delp, S. L. (2014). Changes in tibiofemoral forces due to variations in muscle activity during walking. Journal of Orthopaedic Research, 32, 769-776. doi:10.1002/jor.22601

Ehrig, R. M., Heller, M. O., Kratzenstein, S., Duda, G. N., Trepczynski, A., \& Taylor, W. R. (2011). The SCoRE residual: A quality index to assess the accuracy of joint estimations. Journal of Biomechanics, 44, 1400-1404. doi:10.1016/j.jbiomech.2010.12.009

Ericson, M. O., \& Nisell, R. (1986). Tibiofemoral joint forces during ergometer cycling. The American Journal of Sports Medicine, 14, 285-290. doi:10.1177/036354658601400407

Gerus, P., Sartori, M., Besier, T. F., Fregly, B. J., Delp, S. L., Banks, S. A., ... Lloyd, D. G. (2013). Subjectspecific knee joint geometry improves predictions of medial tibiofemoral contact forces. Journal of Biomechanics, 46, 2778-2786. doi:10.1016/j.jbiomech.2013.09.005

Gosling, C. M., Gabbe, B. J., \& Forbes, A. B. (2008). Triathlon related musculoskeletal injuries: The status of injury prevention knowledge. Journal of Science and Medicine in Sport, 11, 396-406. doi:10.1016/j.jsams.2007.07.009

Hamner, S. R., Seth, A., \& Delp, S. L. (2010). Muscle contributions to propulsion and support during running. Journal of Biomechanics, 43, 2709-2716.doi:10.1016/j.jbiomech.2010.06.025

Holmes, J. C., Pruitt, A. L., \& Whalen, N. J. (1994). Lower extremity overuse in bicycling. Clinics in Sports Medicine, 13, 187-205.

Hunt, M. A., Sanderson, D. J., Moffet, H., \& Timothy Inglis, J. (2003). Biomechanical changes elicited by an anterior cruciate ligament deficiency during steady rate cycling. Clinical Biomechanics, 18, 393-400. doi:10.1016/S0268-0033(03)00046-9

Johnston, T. (2007). Biomechanical Considerations for Cycling Interventions in Rehabilitation. Physical Therapy, 87, 1243-1252. doi:10.2522/ptj.20060210

Leirdal, S., \& Ettema, G. (2011). The relationship between cadence, pedalling technique and gross efficiency in cycling. European Journal of Applied Physiology, 111, 2885-2893. doi:10.1007/s00421011-1914-3

Lin, Y.-C., Dorn, T. W., Schache, A. G., \& Pandy, M. G. (2012). Comparison of different methods for estimating muscle forces in human movement. Proceedings of the Institution of Mechanical Engineers, Part H: Journal of Engineering in Medicine, 226, 103-112. doi:10.1177/0954411911429401

Marshall, R. N., \& McNair, P. J. (2013). Biomechanical risk factors and mechanisms of knee injury in golfers. Sports Biomechanics, 12, 221-230. doi:10.1080/14763141.2013.767371

Menard, M., Domalain, M., Decatoire, A., \& Lacouture, P. (2016). Influence of saddle setback on pedalling technique effectiveness in cycling. Sports Biomechanics, 15, 462-472. doi:10.1080/147 63141.2016.1176244

Neptune, R., \& Kautz, S. (2000). Knee joint loading in forward versus backward pedaling: Implications for rehabilitation strategies. Clinical Biomechanics, 15, 528-535. doi:10.1016/S02680033(00)00005-X

Price, D., \& Donne, B. (1997). Effect of variation in seat tube angle at different seat heights on submaximal cycling performance in man. Journal of Sports Sciences, 15, 395-402. doi:10.1080/026404197367182

Prilutsky, B. I., \& Zatsiorsky, V. M. (2002). Optimization-based models of muscle coordination. Exercise and Sport Sciences Reviews, 30, 32. doi:10.1097/00003677-200201000-00007 
Raabe, M. E., \& Chaudhari, A. M. W. (2016). An investigation of jogging biomechanics using the full-body lumbar spine model: Model development and validation. Journal of Biomechanics, 49, 1238-1243. doi:10.1016/j.jbiomech.2016.02.046

Rhea, M. R. (2004). Determining the magnitude of treatment effects in strength training research through the use of the effect size. The Journal of Strength \& Conditioning Research, 18, 918-920. doi:10.1519/14403.1

Ricard, M. D., Hills-Meyer, P., Miller, M. G., \& Michael, T. J. (2006). The effects of bicycle frame geometry on muscle activation and power during a Wingate anaerobic test. Journal of Sports Science and Medicine, 5, 25-32.

Ruby, P., Hull, M. L., \& Hawkins, D. (1992). Three-dimensional knee joint loading during seated cycling. Journal of Biomechanics, 25, 41-53. doi:10.1016/0021-9290(92)90244-U

Sanderson, D. J., \& Amoroso, A. T. (2009). The influence of seat height on the mechanical function of the triceps surae muscles during steady-rate cycling. Journal of Electromyography and Kinesiology, 19, e465-e471. doi:10.1016/j.jelekin.2008.09.011

Schwameder, H., Lindenhofer, E., \& Müller, E. (2005). Effect of walking speed on lower extremity joint loading in graded ramp walking. Sports Biomechanics, 4, 227-243. doi:10.1080/14763140508522865

Silberman, M., Webner, D., Collina, S., \& Shiple, B. (2005). Road bicycle fit. Clinical Journal of Sport Medicine, 15, 271-276. doi:10.1097/01.jsm.0000171255.70156.da

Spoor, C. W., \& van Leeuwen, J. L. (1992). Knee muscle moment arms from MRI and from tendon travel. Journal of Biomechanics, 25, 201-206. doi:10.1016/0021-9290(92)90276-7

Steele, K. M., DeMers, M. S., Schwartz, M. H., \& Delp, S. L. (2012). Compressive tibiofemoral force during crouch gait. Gait \& Posture, 35, 556-560. doi:10.1016/j.gaitpost.2011.11.023

Tamborindeguy, A. C., \& Rico Bini, R. (2011). Does saddle height affect patellofemoral and tibiofemoral forces during bicycling for rehabilitation? Journal of Bodywork and Movement Therapies, 15, 186191. doi:10.1016/j.jbmt.2009.07.009

Wanich, T., Hodgkins, C., Columbier, J., Muraski, E., \& Kennedy, J. (2007). Cycling injuries of the lower extremity. Journal of the American Academy of Orthopaedic Surgeons, 15, 748-756. doi:10.5435/00124635-200712000-00008

Willy, R. W., Meardon, S. A., Schmidt, A., Blaylock, N. R., Hadding, S. A., \& Willson, J. D. (2015). Changes in tibiofemoral contact forces during running in response to in-field gait retraining. Journal of Sports Sciences, 34, 1602-1611. doi:10.1080/02640414.2015.1125517 